Research Journal of Applied Sciences, Engineering and Technology 6(11): 2041-2044, 2013

DOI:10.19026/rjaset.6.3821

ISSN: 2040-7459; e-ISSN: 2040-7467

(C) 2013 Maxwell Scientific Publication Corp.

$\begin{array}{lll}\text { Submitted: January 08, } 2013 & \text { Accepted: February 22, } 2013 & \text { Published: July 25, } 2013\end{array}$

\title{
Research Article Performance Analysis of Carrier Frequency Offset Estimation Techniques for OFDM Systems
}

\author{
Ishtiaq Akbar and A. Naveed Malik \\ Isra University, Islamabad Campus
}

\begin{abstract}
In this study we will study the effects of Carrier Frequency Offset (CFO) upon Signal to Noise Ratio (SNR) for an OFDM system. We will also present performance analysis of two CFO estimation schemes: time domain Cyclic Prefix (CP) based estimation scheme and frequency domain based Moose scheme, Simulation results show that frequency domain based scheme can achieve better performance in term of Mean Square Error (MSE) than the time domain CP based scheme. Both schemes are analyzed under Doppler fading.
\end{abstract}

Keywords: Carrier Frequency Offset (CFO), Cyclic Prefix (CP), Intercarrier Interference (ICI), Orthogonal Frequency Division Multiplexing (OFDM)

\section{INTRODUCTION}

Orthogonal Frequency Division Multiplexing (OFDM) has gained a great deal of popularity lately due to its high spectral efficiency and robustness to multipath. In the last few years, OFDM has been employed in various commercial applications that include wireless local area networks (IEEE 802.11a and HIPERLAN/2), terrestrial Digital Audio Broadcasting (DABT) and terrestrial digital video broadcasting (DVB-T) (Yi-Hao et al., 2007). It is well known, however, that the performance of OFDM systems is very sensitive to frequency synchronization errors (Jungwon et al., 2004; Pollet et al., 1998). The local oscillators at the transmitter and receiver may not be synchronized and that results in residual Carrier Frequency Offset (CFO) at the receiver after downconversion. Doppler spread, which is present in mobile environments due to changing channel conditions between the transmitter and the receiver, also contributes to the carrier offset. The CFO introduces Inter-Carrier Interference (ICI), which destroys the orthogonally among the subcarriers and attenuates the desired signal, reducing the effective Signal-to-Noise Ratio (SNR) (Pollet et al., 1998). This results in degraded system performance.

To maintain the orthogonality of sub carriers, CFOs and timing errors must be estimated and adequately compensated for. Many methods have been proposed in the literature to estimate and compensate for the CFO. Most of the conventional methods are based on a Frequency-Domain (FD) approach then time domain, where, CFO is estimated with the phase difference between two successive symbols (Kapoor et al., 1998; Xu and Manoakis, 2010). Several schemes
(Morelli and Mengali, 1972, 1999). have been investigated for the CFO estimation in OFDM/OFDMA systems. The methods of CFO estimation can be classified into two groups, i.e., time domain $\mathrm{CFO}$ estimation and frequency domain CFO estimation. Frequency domain CFO estimation schemes can be further classified into two groups ie the training symbol based approache such as (Kapoor et al., 1998) and blind estimation such as Van de Beek et al. (1997). Moose (1994) proposed a pilot based CFO estimation scheme by detecting the phase shifts between several successive identical pilot blocks. Schmidl and Cox (1997) presented a robust synchronization scheme for OFDM using one unique OFDM symbol which has a repetition format within half a symbol period and the acquisition is achieved in two separate steps through the use of a two-symbol training sequence.

This research assesses the effects of CFO upon Signal to Noise Ratio (SNR) of OFDM system and orthoganality of subcarriers. Accordingly research focus on techniques to compensate for carrier frequency offset in OFDM system. Two CFO estimation schemes are analyzed in this regard: The time domain CP based scheme and frequency domain based Moose scheme. Both schemes are analyzed under Doppler fading.

\section{SYSTEM MODEL AND ASSUMPTIONS}

Schematic diagram of Fig. 1 is a baseband equivalent representation of an OFDM system. The input binary data is first fed into a Serial to Parallel $(\mathrm{S} / \mathrm{P})$ converter. Each data stream then modulates the corresponding subcarrier by QPSK or MQAM. Modulations can vary from one subcarrier to another in order to achieve the maximum capacity or the minimum

Corresponding Author: Ishtiaq Akbar, Isra University, Islamabad Campus

This work is licensed under a Creative Commons Attribution 4.0 International License (URL: http://creativecommons.org/licenses/by/4.0/). 


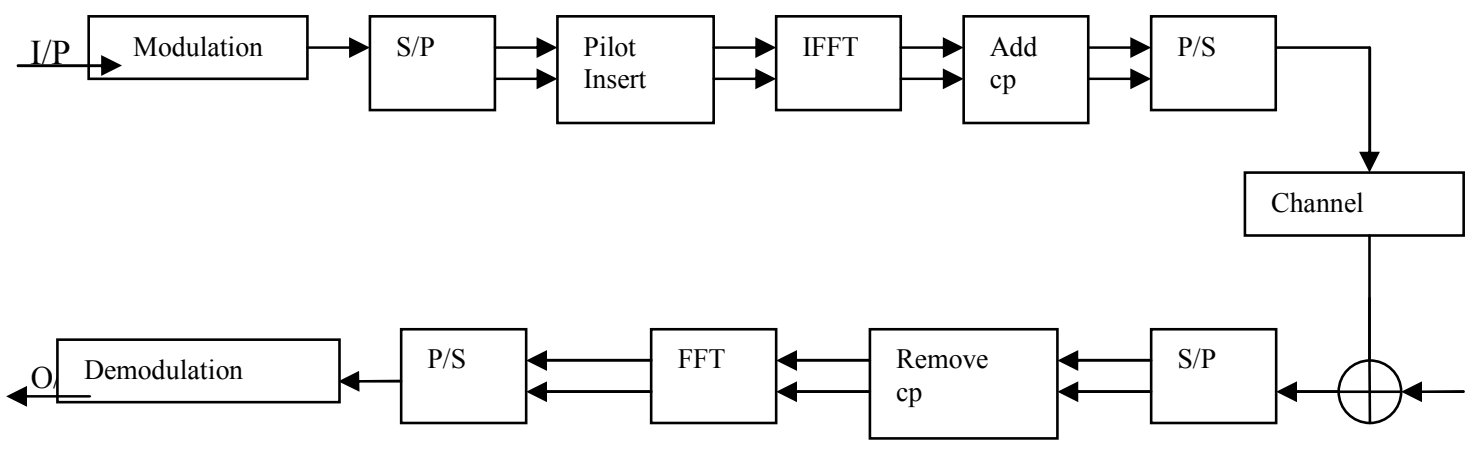

Fig. 1: OFDM baseband model

Bit Error Rate (BER) under various constraints. In this study we use, for simplicity, only QPSK in all the subcarriers and $N$ to denote the number of subcarriers in the OFDM system. The modulated data symbols, represented by complex variables $X(0), \ldots . ., X(N-1)$, are then transformed by the inverse fast Fourier transform (IFFT). The output symbols are denoted as $x(0), \ldots, x(N-1)$. In order to avoid Inter-Symbol Interference (ISI), Cyclic Prefix (CP) symbols, which replicate the rear part of the IFFT output symbols, are added in front of each OFDM symbol. The parallel data are then converted back to a serial data stream before being transmitted over the multipat fading ve channel. The received data $y(0), \ldots \ldots, y(\mathrm{~N}-1)$ corrupted by multipath fading and AWGN are converted back to $Y$ $(0), \ldots \ldots, Y(\mathrm{~N}-1)$ after discarding the prefix and applying FFT and demodulation.

The channel model we adopt in the present study is a multipath slowly time varying fading channel, Which can be described by:

$$
y(k)=\sum_{l=0}^{L-1} h l x(k-l)+z(k) \quad 0 \leq k \leq N-1
$$

where, $h_{l}$ 's $(0 \leq l \leq L-1)$ are Ind-ependent complexvalued Rayleigh fading random variables and $\mathrm{z}_{k}$ 's $(0 \leq k$ $\leq N-1)$ are independent complex-valued Gaussian random variables with zero mean and variance $\sigma^{2} z$ for both real and imaginary components. $L$ is the length of the CIR. In the presence of channel frequency offset, the above equation becomes (Ma et al., 2001):

$$
y(k)=e^{(j 2 \pi k \varepsilon / N)} \sum_{l=0}^{L-1} h l x(k-l)+z(k)
$$

where, $\varepsilon$ is the carrier frequency offset which is normalized by the subcarrier spacing. We assume the frequency acquisition procedure has been completed so that the channel frequency offset is within one half of an interval of the subcarrier spacing, i.e.,

$|\varepsilon| \leq 1$ If the length of the $\mathrm{CP}$ is longer than $L$, there will be no ISI among OFDM symbols. Thus we need to consider only one OFDM symbol with $N$ subcarriers in analyzing the system performance. The system model and performance can be easily extended to the case of multiple symbols. After discarding the cyclic prefix and performing an FFT at the receiver, we can obtain the received data symbol in the frequency domain:

$$
\begin{aligned}
& Y l(k)=\frac{\sin (\pi \varepsilon)}{N \sin \frac{\pi \varepsilon}{N}} X(k) H(k) \sum_{n=0}^{N-1} e^{(j 2 \pi \varepsilon n / N)} \\
& + \text { ICI }(\mathrm{k})+\mathrm{Z}(\mathrm{k})
\end{aligned}
$$

where, $H(m)$ is the frequency response of the channel at subcarrier $m$ and the set of the transformed noise variables $\mathrm{Z}(m), 0 \leq m \leq N-1$ are i.i.d., complex-valued Gaussian variables that have the same distribution as $z(k)$, i.e., with mean zero and variance $\sigma^{2} z$ The noteworthy term in (3) is the $I C I(m)$, which is given as:

$$
I C I(m)=\frac{1}{N} \sum_{k=0}^{N-1} \sum_{n \neq m} X(n) H(n) e^{\left(j 2 \pi \frac{k(n-m+\varepsilon)}{N}\right)}
$$

Eq. (3) shows that the frequency offset degrades the amplitude of the received signal in each subcarrier and introduce Inter-Carrier Interference (ICI). In addition, a common phase shift is introduced to the received signal. That can be used to estimate the frequency offset. In this study we assume the CIR is constant in each OFDM symbol and varies from symbol to symbol according to the fading rate. Furthermore, we assume the system has perfect timing synchronization

\section{TIME DOMAIN CYCLIC PREFIX (CP) BASED CFO ESTIMATION TECNIQUE}

With perfect symbol synchronization, a CFO of $\varepsilon$ results in a phase rotation of $2 \pi \mathrm{n} / \mathrm{N}$ in the received, the phase difference between $\mathrm{CP}$ and the corresponding rear part of an OFDM symbol (spaced $\mathrm{N}$ samples apart) caused by $\mathrm{CFO} \varepsilon$ is $2 \pi \mathrm{N} \varepsilon / \mathrm{N}=2 \pi \varepsilon$. Notice that the samples in the cyclic prefix and their copies, in rear part of OFDM symbol are pair wise correlated. Then, the CFO can be found from the phase angle of the product 
of $\mathrm{CP}$ and the corresponding rear part of an OFDM symbol:

$$
\begin{aligned}
& \hat{\varepsilon}=(1 / 2 \pi) \arg \left\{y_{l}[n] y_{l}[n+N]\right\}, \\
& n=-1, \ldots \ldots . . N g
\end{aligned}
$$

In order to reduce the noise effect, its average can be taken over the samples in a $\mathrm{CP}$ interval as:

$$
\hat{\varepsilon}=(1 / 2 \pi) \arg \left\{\sum_{n=-N g}^{-1} y_{l}[n] y_{l}{ }_{l}[n+N]\right\}
$$

It is obvious from the above equation $\mathrm{CFO}$ estimated in the range of $|\dot{\varepsilon}|<0.5$ integral CFO cannot be estimated by this technique.

\section{FREQUENCY-DOMAIN TECNIQUE FOR CFO ESTIMATION}

If two identical training symbols are transmitted consecutively, the corresponding signals with $\mathrm{CFO}$ of $\varepsilon$ are related with each other as follows:

$$
y_{2}[n]=y_{1}[n] e^{(j 2 \pi \varepsilon)}
$$

Using the above relationship, the CFO can be estimated as:

$$
\hat{\varepsilon}=\frac{1}{2 \pi} \tan ^{-1} \frac{\left\{\sum_{k=0}^{M-1} \operatorname{Im}\left[\mathbf{y}_{1}[k] \mathbf{y}_{2}[k]\right]\right\}}{\left\{\sum_{k=0}^{M-1} \operatorname{Re}\left[\mathbf{y}_{1}[k] \mathbf{y}_{2}[k]\right]\right\}}
$$

Although the range of CFO estimated by Equation is $\left|\varepsilon^{\prime}\right|<0.5$, it can be increased $\mathrm{D}$ times by using a training symbol with $\mathrm{D}$ repetitive patterns.

\section{SIMULATION RESULTS}

Figure 2 shows a $\mathrm{CFO}$ of $\varepsilon$ results in a phase rotation of $2 \pi \varepsilon \mathrm{n} / \mathrm{N}$ in the received signal. Figure 3 gives comparison of BER for different values of CFO. To make a comparison of two schemes, we evaluate the estimation performances over a multipath fading channel. Simulation have been performed by considering system parameters as FFT Size 128, with QPSK modulation technique Fig. 4 give the performance comparisons of the CFO estimation schemes in terms of normalized Mean Square Error

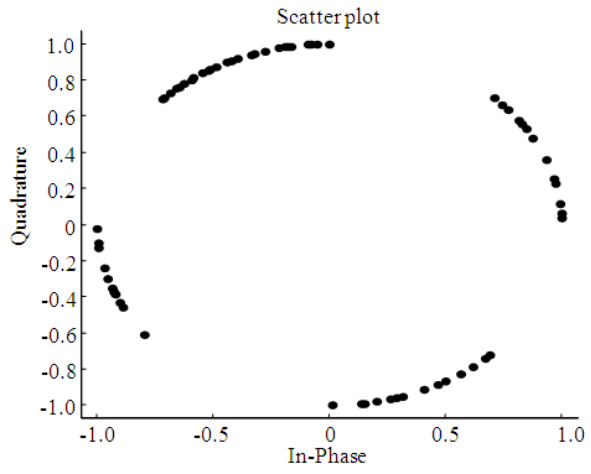

Fig. 2: Constellation of received symbols with $\mathrm{CFO} \varepsilon=0.03$

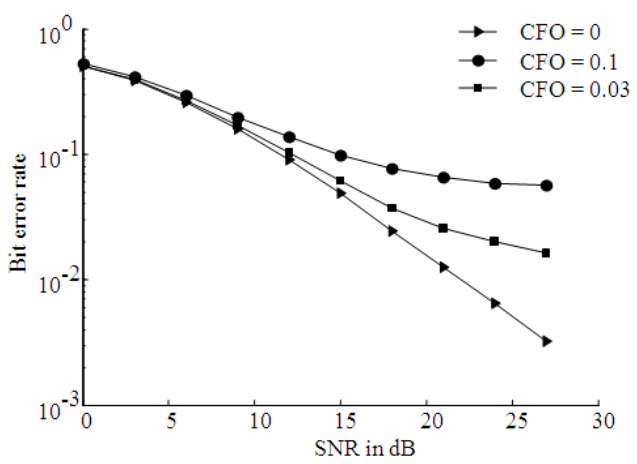

Fig. 3: BER comparison for $\mathrm{CFO} \varepsilon=0,0.03,0.1$

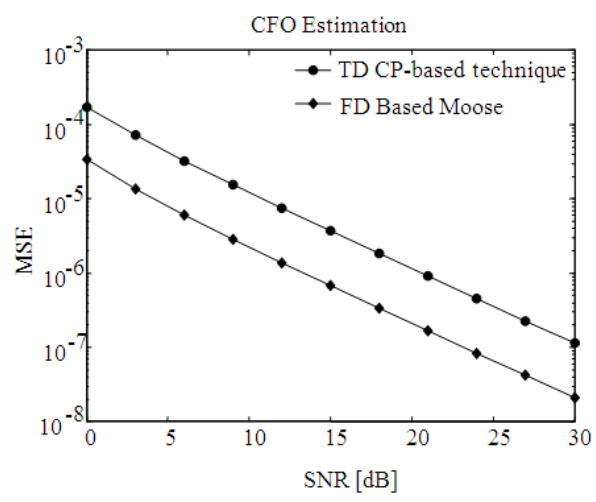

Fig. 4: MSE of CFO estimation technique $\varepsilon=0.03$

(MSE) over multipath fading channel for $\varepsilon=0.03$. The channel has an exponential power delay profile with:

$$
\mathrm{L}=8 E\left\{|h(n)|^{2}\right\}=\frac{1}{\sum_{i=0} e^{-i}} e^{-l} l=0,1 \ldots .7
$$

The frequency domain scheme has better MSE performance then time domain $\mathrm{CP}$ based scheme.

\section{CONCLUSION}

In this study, we investigated the effects of CFO in OFDM system. From mathematical analysis and 
simulation results we see that $\mathrm{CFO}$ introduces InterCarrier Interference (ICI) which destroys the orthogonality of subcarriers.

Two CFO estimation schemes, i.e., the $\mathrm{CP}$ based scheme and frequency domain based Moose scheme have also been applied to the OFDM systems. Simulation results showed that Frequency Domain scheme has better performance than time domain $\mathrm{CP}$ based in terms of normalized MSE over frequency selective channels.

\section{REFERENCES}

Jungwon, L., L. Hui-Ling, D. Toumpakaris and J.M. Cioffi, 2004. Effect of carrier frequency offset on OFDM systems for multipath fading channels. Proceeding of the IEEE Global Telecommunications Conference (GLOBECOM), Marvell Semicond. Inc., Sunnyvale, CA, USA, 6: 3721-3725.

Kapoor, S., D.J. Marchok and Y.F. Huang, 1998. Pilot assisted synchronization for wireless OFDM systems over fast time varying fading channels. Proceeding of 48th Vehicular Technology Conference (VTC), Ottawa, Canada, May.

Ma, X., C. Tepedelenlioglu, G.B. Giannakis and S. Bararossa, 2001. Nondata-aided frequency-offset and channel estimation for OFDM with null subcarriers: Identifiability, algorithms and performance. IEEE J. Sel Area Commun., 19(12): 2504-2515.
Moose, P.H., 1994. A technique for orthogonal frequency division multiplexing frequency offset correction. IEEE Trans. Commun., 42(10): 2908-2914.

Morelli, M. and U. Mengali, 1972. Carrier-frequency estimation for transmissions over selective channels. IEEE Trans. Commun., 48(9): 1580-1589.

Morelli, M. and U. Mengali, 1999. An improved frequency offset estimation for OFDM applications. IEEE Commun. Lett., 3(3): 75-77.

Pollet, T., M.V. Bladel and M. Moeneclaey, 1998. BER sensitivity of OFDM systems to carrier frequency offset and wiener phase noise. IEEE Trans. Comm., 43(2/3/4): 191-193.

Schmidl, T.M. and D.C. Cox, 1997. Robust frequency and timing synchronizationfor OFDM. IEEE Trans. Commun., 45(12): 1613-1621.

Van de Beek, J.J., M. Sandell and P.O. Borjesson, 1997. ML estimation of timing and frequency offset in OFDM systems. IEEE Trans. Signal Process., 45(7): 1800-1805.

$\mathrm{Xu}, \mathrm{W}$. and K. Manoakis, 2010. Robust synchronization for 3GPP LTE system. Proceeding of the IEEE Global Telecommunications Conference (GLOBECOM), Infineon Technol. AG, Neubiberg, Germany, pp: 1-5.

Yi-Hao, P., K. Ying-Chih, L. Gwo-Ruey and W. JyhHorng, 2007. Performance Analysis of a New ICISelf-Cancellation-Scheme in OFDM Systems. IEEE Trans. Consum. Electron., 53: 1333-1338. 